\title{
LETTER \\ Real-Time Object Tracking via Fusion of Global and Local Appearance Models
}

\author{
Ju Hong YOON ${ }^{\dagger}$, Member, Jungho $\mathrm{KIM}^{\dagger}$, and Youngbae HWANG ${ }^{\dagger a}$, Nonmembers
}

\begin{abstract}
SUMMARY In this letter, we propose a robust and fast tracking framework by combining local and global appearance models to cope with partial occlusion and pose variations. The global appearance model is represented by a correlation filter to efficiently estimate the movement of the target and the local appearance model is represented by local feature points to handle partial occlusion and scale variations. Then global and local appearance models are unified via the Bayesian inference in our tracking framework. We experimentally demonstrate the effectiveness of the proposed method in both terms of accuracy and time complexity, which takes $12 \mathrm{~ms}$ per frame on average for benchmark datasets.
\end{abstract}

key words: object tracking, occlusion reasoning, scale estimation, correlation filter

\section{Introduction}

Object tracking is a fundamental problem in computer vision, which can be applied to various applications. Numerous tracking algorithms have been addressed various problems regarding appearance changes such as partial occlusion, scale variation, illumination changes, and pose variation to retain the robustness of visual tracking. It is possible to categorize existing tracking algorithms into two groups according to their appearance modeling as local and global representations [1]. The global representation of an object is robust against noise and background clutters due to the global geometric constraint and is computationally efficient, especially when we design a discriminative model. Among various methods that are based on the global representation, the correlation filter has been successfully applied to object tracking because of its computational efficiency in representing global statistical characteristic of object appearance against background [4], [5] despite of its limitation on scale variation and partial occlusion as shown in Fig. 1. On the contrary, the local representation captures the local structure of an object [3] so that they are robust against partial occlusion and scale variation but they are weak to cluttered scenes [1]. In this context, we propose a fusion framework for robust and fast object tracking in consideration of complementary characteristics of local and global representations; hence, two representations are unified via the Bayesian inference. The overall procedure is illustrated in Fig. 2.

\footnotetext{
Manuscript received April 7, 2017.

Manuscript revised July 19, 2017.

Manuscript publicized August 7, 2017.

${ }^{\dagger}$ The authors are with Korea Electronics Technology Institute KETI, Korea.

a) E-mail: ybhwang@keti.ac.kr

DOI: 10.1587/transinf.2017EDL8077
}

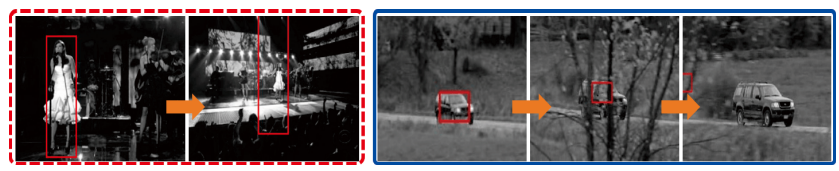

Fig. 1 Tracking failure cases caused by scale variation (the dashed red box) and occlusion (the solid blue box)

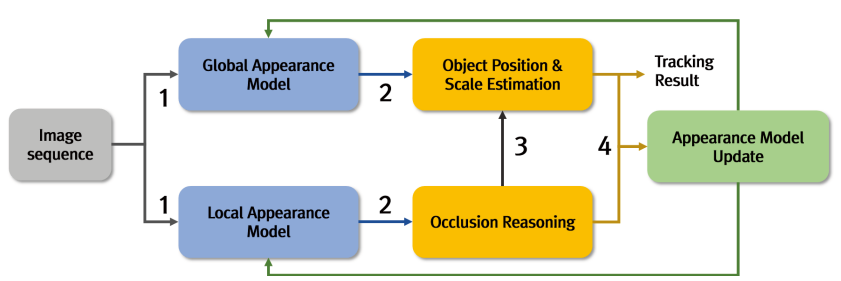

Fig. 2 Overall framework of the proposed tracking algorithm: each number denotes the processing sequence.

\section{Bayesian Framework for Object Tracking}

In the Bayesian approach, we estimate an object state $\mathbf{x}_{t}$ which maximizes the posterior probability,

$$
\hat{\mathbf{x}}_{t}=\arg \max _{\mathbf{x}_{t}} p\left(\mathbf{x}_{t} \mid \mathbf{z}_{1: t}\right),
$$

where observations up to frame $t$ is represented by $\mathbf{z}_{1: t}=$ $\left\{\mathbf{z}_{1}, \ldots, \mathbf{z}_{t}\right\}$. Here, we define an object state as $\mathbf{x}_{t}=$ $\left[u_{t}, v_{t}, s_{t}\right]^{\top}$ where $\left(u_{t}, v_{t}\right)$ denotes the center of an object where the object is represented as a bounding box. $s_{t}$ denotes the scale of the object by assuming a constant aspect ratio over time. $\mathbf{z}_{t}$ is an image at time $t$. We formulate the posterior probability as

$$
\begin{aligned}
& p\left(\mathbf{x}_{t} \mid \mathbf{z}_{1: t}\right) \\
& \propto p_{o c c}\left(\mathbf{z}_{t} \mid \mathbf{x}_{t}\right) \int p\left(\mathbf{x}_{t} \mid \mathbf{x}_{t-1}\right) p\left(\mathbf{x}_{t-1} \mid \mathbf{z}_{1: t-1}\right) d \mathbf{x}_{t-1},
\end{aligned}
$$

where the transition density $p\left(\mathbf{x}_{t} \mid \mathbf{x}_{t-1}\right)$ follows a random walk motion. To handle occlusion, we first design the likelihood with occlusion $p_{\text {occ }}\left(\mathbf{z}_{t} \mid \mathbf{x}_{t}\right)$ as

$$
\begin{aligned}
& p_{\text {occ }}\left(\mathbf{z}_{t} \mid \mathbf{x}_{t}\right) \\
& =P_{\text {occ }}\left(\mathbf{z}_{t}\right) p_{m}\left(\mathbf{x}_{t}\right)+\left(1-P_{\text {occ }}\left(\mathbf{z}_{t}\right)\right) p\left(\mathbf{z}_{t} \mid \mathbf{x}_{t}\right),
\end{aligned}
$$

where $P_{\text {occ }}\left(\mathbf{z}_{t}\right)$ is an occlusion indicator and $p_{m}\left(\mathbf{x}_{t}\right)$ is the prior spatial transition of an object. Here, we set $P_{o c c}\left(\mathbf{x}_{t}\right)$ to 1 if partial or full occlusion occurs and 0 otherwise. This occlusion is determined by computing the learning rate in (11). 
The likelihood distribution only depends on the prior spatial transition of an object, i.e., $p_{m}\left(\mathbf{x}_{t}\right)$, if the object is likely to be occluded. Otherwise, the likelihood $p\left(\mathbf{z}_{t} \mid \mathbf{x}_{t}\right)$ in (3) is defined based on two appearance models,

$$
p\left(\mathbf{z}_{t} \mid \mathbf{x}_{t}\right)=p_{G}\left(\mathbf{z}_{t} \mid \mathbf{x}_{t}\right) p_{L}\left(\mathbf{z}_{t} \mid \mathbf{x}_{t}\right) .
$$

Here, $p_{G}\left(\mathbf{z}_{t} \mid \mathbf{x}_{t}\right)$ in (7) and $p_{L}\left(\mathbf{z}_{t} \mid \mathbf{x}_{t}\right)$ in (9) denote likelihood distributions of global and local representations, respectively. Since the position and the scale of the object are independent of each other, we independently obtain the position and the scale that maximize the likelihood of the global and local appearance models. In this work, using the global appearance model, we estimate the object position in the image coordinate, and we measure the scale variation using the local appearance model. Their formulation are explained in detail in the next section.

\section{Global and Local Appearance Modeling}

To train the global appearance model, conventional tracking approaches utilize positive and negative training samples extracted from predefined regions [11], [12]. Different from the previous approaches, the correlation filter enables dense sampling based on convolution process whose computational complexity is dramatically reduced by the simple element-wise product in the frequency domain. An appearance model $\mathbf{h}_{t}$ (a correlation filter) at time $t$ satisfies a convolution process $\mathbf{r}_{t} * \mathbf{h}_{t}=\mathbf{g}$ where $\mathbf{r}_{t}$ is an image template of the region of interest and $\mathbf{g}$ is a predefined two-dimensional Gaussian distribution centered at the bounding box of an object. This process is converted into the element-wise product in the frequency domain as $\mathcal{F}\left(\mathbf{h}_{t}\right) \odot \mathcal{F}\left(\mathbf{r}_{t}\right)=\mathcal{F}(\mathbf{g})$. We adopt the correlation filter proposed in [4] which utilizes the Gaussian kernel $\kappa(a, b)$ as

$$
\begin{aligned}
& \kappa(a, b) \\
& =\exp \left(-\frac{1}{a^{2}}\left(\|a\|^{2}+\|b\|^{2}-2 \mathcal{F}^{-1}\left(\mathcal{F}(a) \odot \mathcal{F}^{*}(b)\right)\right)\right),
\end{aligned}
$$

where $a$ and $b$ represent an image template, respectively. With this kernel, the appearance model is computed by

$$
\mathbf{h}_{t}=\mathcal{F}^{-1}\left(\frac{\mathcal{F}(\mathbf{g})}{\mathcal{F}\left(\kappa\left(\mathbf{r}_{t}, \mathbf{r}_{t}\right)+\lambda\right)}\right),
$$

where $\lambda$ denotes the regularization term. Based on the appearance model $\mathbf{h}_{t}$, the likelihood of the global representation is calculated by

$$
p_{G}\left(\mathbf{z}_{t} \mid \mathbf{x}_{t}\right)=\mathcal{F}^{-1}\left(\mathcal{F}\left(\mathbf{h}_{t}\right) \odot \mathcal{F}\left(\kappa\left(\mathbf{z}_{t}, \mathbf{r}_{t}\right)\right)\right),
$$

As mentioned in the introduction, we do not use the local feature points to find the object global position since their reliability is individually very low under object appearance changes. Therefore, we only indirectly utilize the local feature points to measure object scale changes due to its flexibility.

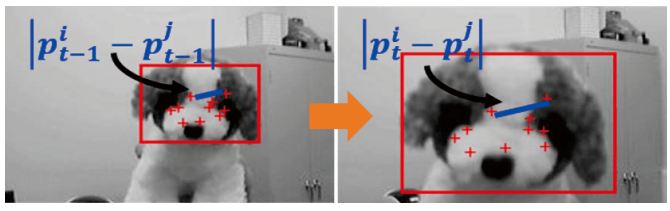

Fig. 3 Scale estimation: the relative distance between two points increases proportional to the object size.

Similar to the global appearance model, the local appearance model is also assumed to follow the Gaussian distribution. We estimate the scale variation. Hence, its likelihood function is defined by an 1-dimensional Gaussian distribution with the mean $\bar{s}_{t}$ and the variance $\sigma$ as follow.

$$
p_{L}\left(\mathbf{z}_{t} \mid \mathbf{x}_{t}\right)=\mathcal{N}\left(s_{t} ; \bar{s}_{t}, \sigma\right) .
$$

Based on the Gaussian distribution, we can maximize the above likelihood with the mean $\bar{s}_{t}$. In this work, we compute $\bar{s}_{t}$ by exploiting a local structure of object appearance based on geometric relationships of pairwise feature points as shown in Fig. 3. In the initial frame, we first extract the local feature points in the object bounding box using the Harris corner detector [6] to construct the geometric structure of the object appearance which formulates the local appearance model. Hence, the local appearance model contains a set of those feature points (i.e., center positions) at frame $t$, which is denoted by $\mathcal{P}_{t}=\left\{\mathbf{p}_{t}^{i} \mid i \in \mathcal{I}_{t}\right\}$ where $\mathcal{I}_{t}$ includes feature point index $i$, and feature point $\mathbf{p}_{t}^{i}$ is represented by the pixel position as $\mathbf{p}_{t}^{i}=\left[u_{t}^{i}, v_{t}^{i}\right]^{\top}$. We sequentially track those feature points at each frame by using the KLT tracker [2], and then from the geometric difference of pairs of feature points between frame $t-1$ and $t$, the mean of the scale variation is calculated by

$$
\bar{s}_{t}=\frac{1}{D} \sum_{i \in \mathcal{I}_{t}} \sum_{j \in \mathcal{I}_{t}, i \neq j} \frac{\left\|\mathbf{p}_{t}^{i}-\mathbf{p}_{t}^{j}\right\|}{\left\|\mathbf{p}_{t-1}^{i}-\mathbf{p}_{t-1}^{j}\right\|},
$$

where $D=N_{t}\left(N_{t}-1\right)$ and $N_{t}$ is the number of feature points that survive at frame $t$.

Remark: Similar to the previous correlation filter-based tracker [4], we also fix the size of the object bounding box to compute the likelihood of the global appearance and to update the global appearance model due to its efficiency. For this reason, to reflect the size variations in the tracking and the update steps, we resize the region of interest (ROI) of an image with the inverse of the scale variation $\bar{s}_{t}$ as $\mathrm{ROI}=\operatorname{resize}\left(\mathrm{ROI}, \frac{1}{\bar{s}_{t}}\right)$. By doing this, we can keep the search region and the appearance model size. On the contrary, to display the tracking result on the image, we resize the bounding box $(B B)$ proportional to the scale variation as $\tilde{\mathrm{BB}}=\operatorname{resize}\left(\mathrm{BB}, s_{t}\right)$. This proportionally resized $\mathrm{BB}$ is used as tracking results for the performance evaluation.

\section{Occlusion-Aware Appearance Update}

When object appearance gradually changes, we need to update the appearance model to deal with its variations with 


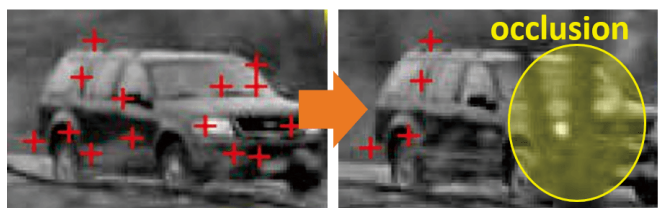

Fig. 4 Occlusion reasoning by feature points: Most of feature points disappear as the car is occluded by the tree.

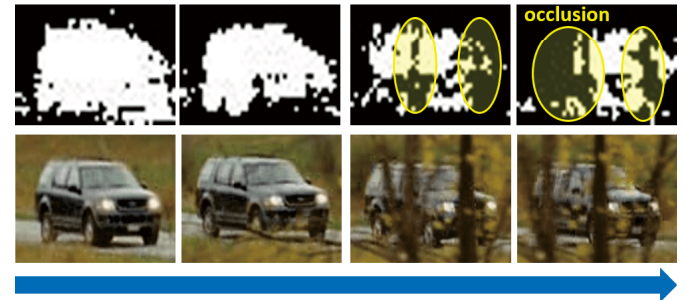

Fig.5 Occlusion reasoning by color pixel: when occlusion occur, the region of the object pixels is reduced.

time. To prevent updating the appearance model from occluded parts, we propose a simple but robust occlusion reasoning method. In the proposed method, we utilize a set of local feature points within the object appearance and color histograms for both the background and the target object. First, occlusion reasoning can be achieved by computing the number of tracked features between frame $t-1$ and $t$. Since there are severe appearance changes when occlusion occurs, a local tracker fails to track feature points as shown in Fig. 4. For occlusion reasoning, we first compute the ratio of tracked points as $R^{p}=N_{t}^{p} / N_{t-1}^{p}$.

Since local features are likely to be falsely tracked due to cluttered scenes and image noise, we additionally utilize color histograms. To reduce inclusion of the background color in the histogram model, we first extract the color pixels from the set of feature points $\mathcal{P}_{t}^{f}=\left\{\mathbf{p}_{t}^{i} \mid i \in \mathcal{I}_{t}\right\}$ used in the local appearance model, and then we obtain the $q$-bin foreground histogram model $\mathcal{H}_{t}^{f}\left(\mathcal{P}_{t}^{f}, q\right)$ by doing quantization and counting the color pixels in each quantized bin. Similarly, we obtain the background histogram model as $\mathcal{H}_{t}^{b}\left(\mathcal{P}_{t}^{b}, q\right)$ and $\mathcal{P}_{t}^{b}$ is the set of feature points collected from the background region. When a pixel $\mathbf{c}$ is given, we obtain a corresponding likelihood as

$$
L^{h}(\mathbf{c})=\frac{\mathcal{H}_{t}^{f}\left(\mathcal{P}_{t}, q\right)[\mathbf{c}]}{\mathcal{H}_{t}^{f}\left(\mathcal{P}_{t}, q\right)[\mathbf{c}]+\mathcal{H}_{t}^{b}\left(\mathcal{P}_{t}^{b}, q\right)[\mathbf{c}]} .
$$

We set the label of the pixel $c, I(c)$ to 1 if $L^{h}(c)>\beta$ and 0 , otherwise. We set $\beta=0.7$. The result of pixel-wise occlusion detection is given in Fig. 5. Then we count the pixels belonging to the non-occluded parts as [7] $N_{t}^{h}=\sum_{c \in T} I(c)$. The ratio of non-occluded pixels is defined by $R_{t}^{h}=N_{t}^{h} /|T|$ where $|T|$ represents the number of pixels within the image template.

We determine the learning rate $\tau$ and an occlusion event by using the ratio of survived feature points $R_{t}^{p}$ and the ratio of non-occluded pixels $R_{t}^{h}$ as

$$
\tau=r \times \max \left\{\left((1-w) R_{t}^{p}+w R_{t}^{h}-\eta\right), 0\right\} .
$$

Here, we assume that if the weighted sum of two ratios is greater than the margin $\eta=0.5$, then this object undergoes severe occlusion. The fusion weight $w=0.5$ combines two ratios. Therefore, we equally fuse two terms. The variable $r$ representing the bound parameter is set to 0.2 in our work. We update the appearance model with the new appearance using the learning rate $\tau$ whose maximum value is bounded by 0.1 with $r=0.2$. We assume that when $\tau=0$, occlusion occurs. Therefore, the occlusion event $P_{o c c}\left(\mathbf{z}_{t}\right)$ in (3) is also set to 0 , and we do not correct the object state with a current observation. Otherwise, $P_{\text {occ }}\left(\mathbf{z}_{t}\right)=1$, and we update the global appearance model by

$$
\begin{aligned}
& \mathbf{h}_{t+1}=\mathcal{F}^{-1}\left((1-\tau) \mathcal{F}\left(\mathbf{h}_{t}\right)+\tau \frac{\delta \mathcal{F}(\mathbf{g})}{\mathcal{F}\left(\kappa\left(\hat{\mathbf{r}}_{t}, \hat{\mathbf{r}}_{t}\right)\right)+\lambda}\right), \\
& \mathbf{r}_{t+1}=(1-\tau) \mathbf{r}_{t}+\tau \hat{\mathbf{r}}_{t},
\end{aligned}
$$

where $\hat{\mathbf{r}}$ is an image template of the region of interest whose center and width are defined by a center and the twice enlarged size of an estimated object.

\section{Experiments}

The proposed tracker was implemented using MATLAB, and it operates approximately $12 \mathrm{~ms}$ per frame with a single core processor. The approximate runtime of each module is summarized as (i) position tracking: $6 \mathrm{~ms}$ (ii) scale estimation: $4 m s$ (iii) occlusion reasoning: $0.8 m s$ (iv) the appearance update: $1 \mathrm{~ms}$ We evaluated the proposed tracking algorithm on twelve challenging benchmark videos [8]. Those selected sequences contain severe scale variation or occlusion, and both events or problems simultaneously occur.

Parameters. We empirically set a threshold $\beta=0.7$ for $L^{h}(c)$ below (10). Except for $\beta$, we determined other parameter values in (11) according to following reasons. We set $\eta=0.5$ because when the object undergoes severe occlusion, at least the half of feature points disappear or at least the half of pixels severely change. The equal fusion weight $w=0.5$ does not critically affect tracking performance as shown in Table 3 because both terms well measure occlusions. Therefore, it is reasonable to fuse them with equal weights. As similar to the previous well-known approaches [4], [5], [9], a typically used value for the learning rate is about $\tau=0.05 \sim 1.0$ when we update the appearance incrementally in online object tracking. For this reason, we set $r=0.2$; therefore, the maximum learning rate $\tau$ is bounded by 0.1 . We tested that how much our tracker is affected by the parameter setting as shown in Tables 1 4. In each trial, we changed one parameter value and fixed others to measure the tracking performance using two metrics, i.e., the average of pixel distance errors and the average of overlap ratios. Note that we did not set unreasonable parameter values in experiments. The experimental results with Tables 1 - 4 validate that when the reasonable parameters are roughly set, our tracker will robustly show similar 
Table 1 Performance evaluations on different $\beta$ below (10).

\begin{tabular}{|c|c|c|c|c|}
\hline $\operatorname{metric} \beta$ & 0.5 & 0.6 & 0.7 & 0.8 \\
\hline distance & 12.63 & 10.97 & 9.73 & 11.27 \\
\hline overlap & 0.59 & 0.63 & 0.64 & 0.58 \\
\hline
\end{tabular}

Table 2 Performance evaluations on different $\eta$ in (11).

\begin{tabular}{|c|c|c|c|c|}
\hline metric $\eta$ & 0.3 & 0.4 & 0.5 & 0.6 \\
\hline distance & 12.04 & 9.62 & 9.73 & 12.51 \\
\hline overlap & 0.61 & 0.62 & 0.64 & 0.61 \\
\hline
\end{tabular}

Table 3 Performance evaluations on different $w$ in (11).

\begin{tabular}{c|c|c|c|c}
\hline metric & 0.4 & 0.5 & 0.6 & 0.7 \\
\hline distance & 9.91 & 9.73 & 8.74 & 11.35 \\
overlap & 0.65 & 0.64 & 0.63 & 0.62 \\
\hline
\end{tabular}

Table 4 Performance evaluations on different $r$ in (11).

\begin{tabular}{c|c|c|c}
\hline metric & $r$ & 0.1 & 0.2 \\
\hline distance & 10.63 & 9.73 & 10.13 \\
overlap & 0.62 & 0.64 & 0.58 \\
\hline
\end{tabular}

Table 5 The average center location error (pixel): a lower value is better.

\begin{tabular}{c|c|c|c|c}
\hline Sequence & MFT [3] & KCF [4] & CF2 [13] & proposed \\
\hline Car4 & 285.35 & 19.13 & 7.04 & 4.78 \\
CarScale & 243.72 & 117.56 & 21.82 & 8.40 \\
Dog1 & 15.04 & 72.41 & 4.72 & 5.34 \\
Polarbear & 29.38 & 14.37 & 10.45 & 11.63 \\
Singer1 & 8.45 & 153.92 & 9.16 & 3.19 \\
Sphere & 30.16 & 11.25 & 5.61 & 11.05 \\
Coke & 7.72 & 7.52 & 6.93 & 6.18 \\
David & 13.89 & 16.58 & 6.19 & 4.46 \\
Freeman1 & 48.74 & 124.45 & 8.37 & 24.71 \\
Girl & 89.19 & 37.34 & 10.43 & 17.30 \\
Sylv & 29.60 & 11.54 & 10.63 & 8.93 \\
Tiger1 & 33.72 & 25.35 & 10.11 & 10.73 \\
\hline Average & 76.49 & 50.95 & 9.29 & 9.73
\end{tabular}

performance.

We compared the proposed tracker with the most related methods, i.e., the median flow tracker (MFT), which is the KLT-based tracker [3] and the kernelized correlation filter (KCF), which is the state-of-the-art spatial tracker [4]. The MFT estimates the bounding box of the target object based on local appearance model, and the KCF utilizes the correlation filter as a global appearance model.

Quantitative comparison. We present an average of a center location error and an average of an overlap [10] between the ground truth and tracking results. These two metrics are widely used to evaluate tracking performance [10], [11]. We summarize the performance of tracking methods in Tables 5 and 6 . According to Tables 5 and 6 , the overall performance of the proposed tracker is better than that of the MFT and KCF because the proposed tracker efficiently exploits the advantages of the local appearance model to estimate scale variation and occlusion estimation and the ro-
Table 6 The average overlap: a higher value is better.

\begin{tabular}{c|c|c|c|c}
\hline Sequence & MFT [3] & KCF [4] & CF2 [13] & proposed \\
\hline Car4 & 0.15 & 0.47 & 0.73 & 0.82 \\
CarScale & 0.26 & 0.38 & 0.49 & 0.63 \\
Dog1 & 0.49 & 0.46 & 0.78 & 0.77 \\
Polarbear & 0.49 & 0.57 & 0.48 & 0.64 \\
Singer1 & 0.65 & 0.16 & 0.71 & 0.84 \\
Sphere & 0.51 & 0.69 & 0.84 & 0.69 \\
Coke & 0.56 & 0.53 & 0.58 & 0.60 \\
David & 0.55 & 0.52 & 0.74 & 0.59 \\
Freeman1 & 0.26 & 0.24 & 0.43 & 0.28 \\
Girl & 0.20 & 0.52 & 0.75 & 0.65 \\
Sylv & 0.35 & 0.60 & 0.64 & 0.62 \\
Tiger1 & 0.26 & 0.40 & 0.57 & 0.56 \\
\hline Average & 0.39 & 0.46 & 0.65 & 0.64 \\
\hline
\end{tabular}

bustness of the global appearance model under illumination and contrast variations. As shown in Tables $5-6$, the KCF favorably tracks the objects in the Car4, the Polarbear, the Sphere, the Coke, and the Sylv sequences where relatively small scale variations occur. However, in the CarScale, the Dog1, the Singer1, and the Girl, objects undergo large scale variations where the KCF shows limited performance compared to the proposed tracker. The MFT tracks objects robustly in the Singer1 and the Dog1 where only the size of objects changes significantly with acceptable illumination and pose variations. However, the MFT fails to track local features in the Car4, the CarScale, the Freeman1, and the Girl due to low contrast, abrupt illumination changes, and occlusion. As a result, the MFT fails to track the objects. Different from the MFT, the global appearance model of the proposed tracker handles those appearance variation.

Qualitative comparison. The robustness of the proposed tracker under scale variations and occlusion is also demonstrated with Figs. 6, 7, and 8. As shown in Fig. 6, the proposed tracker and the MFT more accurately estimate the object size and position in comparison with the KCF because the KCF does not consider the scale variation in tracking. As shown in Fig. 7, the proposed tracker more robustly track the objects than the KCF and the MFT because it can deal with low contrast by using the global appearance model. Compared to other test videos, the CarScale contains long-term scale variations and partial occlusions as shown in Fig. 8. The proposed tracker more robustly tracked the objects than the KCF and the MFT trackers because the proposed tracker can deal with visual ambiguity from cluttered scene by using the global appearance model, and it can overcome significant scale variation by using the proposed scale estimation based on the local appearance model.

Recently, image features generated by deep learning techniques are widely used in object tracking due to their high performance. Although we propose a different concept of tracking approaches, we illustrate the advantages and disadvantages by comparing one of deep feature-based tracker (i.e., CF2) [13], which is also formulated based on the correlation filter. The results of the $\mathrm{CF} 2$ were obtained by using the open source code provided by the author. As shown in Tables 5-6, both trackers show similar performance in overall. In the CarScale where occlusions and scale vari- 

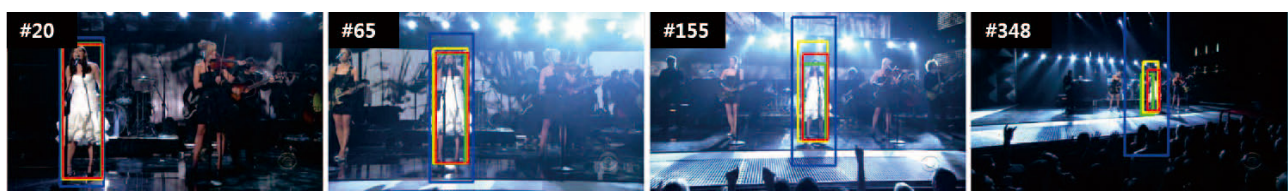

Fig. 6 Singer1: Scale and illumination variation occur (frame \#20, \#65, \#155, \#348). Red (the proposed tracker), blue (KCF), green (MFT), yellow (CF2).
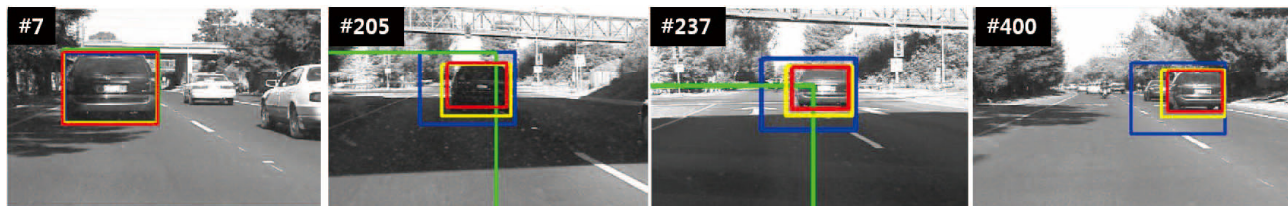

Fig. 7 Car4: Scale variation with low contrast occur (frame \#7, \#205, \#237, \#400). Red (the proposed tracker), blue (KCF), green (MFT), yellow (CF2).
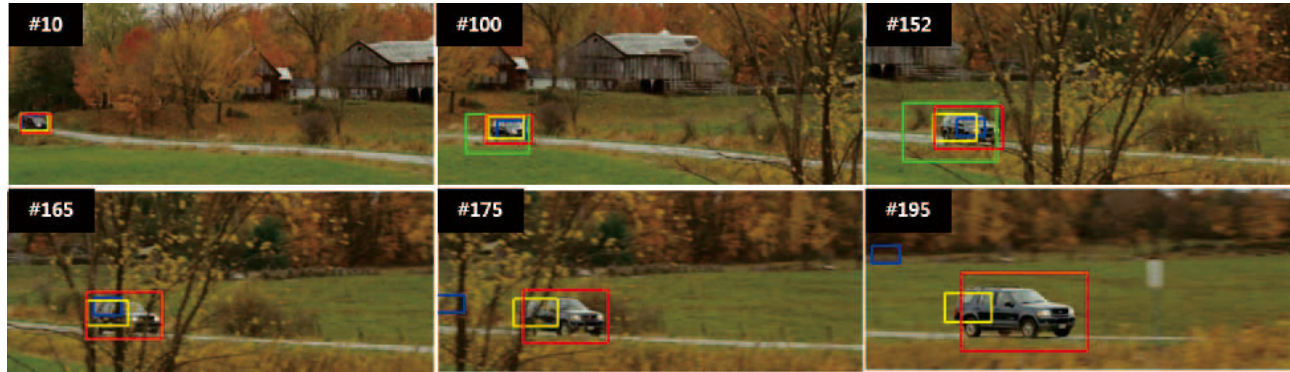

Fig. 8 CarScale: Scale variation occurs in the entire frames, and the car undergoes occlusion from frame \#152 to frame \#180. Red (the proposed tracker), blue (KCF), green (MFT), yellow (CF2).

ations are severe, the proposed tracker show better performance, and in the Car4 and the Singer1 where only scale changes are prominent, the proposed tracker shows more favorable performance in terms of the overlap in Table 6 and in Figs. 6 - 8. Compared with our tracker, the CF2 more robustly handles the severe appearance changes, especially for low contrast and severe pose variation. In terms of computational complexity, our tracker achieves approximately 83 fps. However, the CF2 takes $2 \mathrm{fps}$ due to the high dimensional feature. Our tracker is approximately 40 times faster than the $\mathrm{CF} 2$.

\section{Conclusion}

We proposed a fusion framework which integrates a global appearance with a local structure of the object appearance for real-time object tracking. To achieve the efficient fusion of complementary characteristics of two representations, scale and occlusion reasoning are introduced, and these cues are incorporated into the Bayesian framework. The experimental results demonstrate the improved performance in various tracking environments.

\section{Acknowledgments}

This research was supported by the "Cross-Ministry Giga KOREA Project" of the Ministry of Science, ICT and Future Planning, Republic of Korea (ROK), [GK17C0100].

\section{References}

[1] X. Li, W. Hu, C. Shen, Z. Zhang, A. Dick, and A. van den Hengel, "A survey of appearance models in visual object tracking," ACM Trans. Intell. Systems and Technology, vol.4, no.4, 2013.

[2] J. Shi and C. Tomasi, "Good features to track," IEEE Conf. Computer Vision and Pattern Recognition, pp.593-600, 1994.

[3] Z. Kalal, K. Mikolajczyk, and J. Matas, "Forward-backward error: automatic detection of tracking failures," Int. Conf. Pattern Recognition, pp.2756-2759, 2010.

[4] J.F. Henriques, R. Caseiro, P. Martins, and J. Batista, "High-speed tracking with kernelized correlation filters," IEEE Trans. Pattern Anal. Mach. Intell., vol.37, no.2, pp.583-596, 2015.

[5] D.S. Bolme, J.R. Beveridge, B.A. Draper, and Y.M. Lui, "Visual object tracking using adaptive correlation filters," IEEE Conference on Computer Vision and Pattern Recognition, pp.2544-2550, 2010.

[6] C. Harris and M. Stephens, "A combined corner and edge detector," Proc. Fourth Alvey Vision Conference, pp.23.1-23.6, 1988.

[7] H. Possegger, T. Mauthner, and H. Bischof, "In defense of colorbased model-free tracking," IEEE Conf. Computer Vision and Pattern Recognition, pp.2113-2120, 2015.

[8] Y. Wu, J. Lim, and M.-H. Yang, "Online object tracking: a benchmark," IEEE Conference on Computer Vision and Pattern Recognition, pp.2411-2418, 2013.

[9] D.A. Ross, J. Lim, R.-S. Lin, and M.-H. Yang, "Incremental learning for robust visual tracking," International Journal of Computer Vision, vol.77, no.1, pp.125-141, 2008.

[10] J. Santner, C. Leistner, A. Saffari, T. Pock, and H. Bischof, "Parallel robust online simple tracking," IEEE Conf. Computer Vision and Pattern Recognition, pp.723-730. 2010.

[11] B. Babenko, M.-H. Yang, and S. Belongie, "Robust object tracking 
with online multiple instance learning," IEEE Trans. Pattern Anal. Mach. Intell., vol.33, no.8, pp.1619-1632, 2011.

[12] H. Grabner, M. Grabner, and H. Bischof, "Real-time tracking via on-line boosting," Proceedings of the British Machine Vision Conference, pp.6.1-6.10, 2006.
[13] C. Ma, J.-B. Huang, X. Yang, and M.-H. Yang, "Hierarchical convolutional features for visual tracking," IEEE International Conference on Computer Vision, pp.3074-3082, 2015. 